\title{
Air-Guiding Photonic Bandgap Fibers: Spectral Properties, Macrobending Loss, and Practical Handling
}

Hansen, Theis Peter; Broeng, Jes; Jakobsen, Christian; Vienne, Guillaume; Simonsen, Harald R.;

Nielsen, Martin Dybendal; Skovgaard, Peter M.W.; Folkenberg, Jacob Riis; Bjarklev, Anders Overgaard

Published in:

Journal of Lightwave Technology

Link to article, DOI:

10.1109/JLT.2003.822833

Publication date:

2004

Document Version

Publisher's PDF, also known as Version of record

Link back to DTU Orbit

Citation (APA):

Hansen, T. P., Broeng, J., Jakobsen, C., Vienne, G., Simonsen, H. R., Nielsen, M. D., Skovgaard, P. M. W., Folkenberg, J. R., \& Bjarklev, A. O. (2004). Air-Guiding Photonic Bandgap Fibers: Spectral Properties, Macrobending Loss, and Practical Handling. Journal of Lightwave Technology, 22(1), 11-15.

https://doi.org/10.1109/JLT.2003.822833

\section{General rights}

Copyright and moral rights for the publications made accessible in the public portal are retained by the authors and/or other copyright owners and it is a condition of accessing publications that users recognise and abide by the legal requirements associated with these rights.

- Users may download and print one copy of any publication from the public portal for the purpose of private study or research.

- You may not further distribute the material or use it for any profit-making activity or commercial gain

- You may freely distribute the URL identifying the publication in the public portal 


\title{
Air-Guiding Photonic Bandgap Fibers: Spectral Properties, Macrobending Loss, and Practical Handling
}

\author{
Theis P. Hansen, Jes Broeng, Christian Jakobsen, Guillaume Vienne, Harald R. Simonsen, Martin D. Nielsen, \\ Peter M. W. Skovgaard, Jacob R. Folkenberg, and Anders Bjarklev
}

\begin{abstract}
For development of hollow-core transmission fibers, the realizable fibers lengths, bandwidth, characterization, and compatibility with standard technology are important issues. We report record-length air-guiding fiber, spectral properties, splicing, and optical time domain reflectometer (OTDR) measurements. Furthermore, spectral macrobending loss measurements for two different designs of air-core photonic bandgap fibers are presented. While bending loss is observed, it does not limit operation for all practical bending diameters $(>5 \mathrm{~mm})$.
\end{abstract}

Index Terms-Optical fiber losses, optical fiber measurements, optical fibers, photonic bandgap (PBG) fibers, photonic crystal fibers (PCFs).

\section{INTRODUCTION}

$\mathbf{G}$ REAT interest has recently been shown for photonic bandgap (PBG) fibers that carry most of the field intensity in an air (or hollow) core [1]-[3]. The reason for this interest in air-guiding PBG fibers is their potential of alleviating a number of barriers of conventional optical fibers in terms of attenuation, nonlinearities and available transmission windows. In order for air-guiding PBG fibers to fulfill the potential of the aforementioned aspects, it is a requirement that long fiber lengths can be fabricated with low loss, that the fibers can be made compatible with standard optical fiber technology, and that their spectral properties are well understood. While initial loss values in these fibers were prohibitive for many applications, the recent report of a fiber with $13 \mathrm{~dB} / \mathrm{km}$ attenuation [3] clearly highlights the potential of the technology. One important fiber property is macrobending loss. Macrobending loss measurements in index-guiding photonic crystal fibers (PCFs) demonstrating unusual bending loss properties, with a bend loss edge at short wavelengths were presented in [4]. Early theoretical work has indicated air-guiding fibers to be highly bending insensitive [5]. Possible applications of air-guiding PCFs include high-power delivery for medical use, material processing, etc. For such applications, fibers with low bending

Manuscript received June 30, 2003; revised October 13, 2003. The work of T. P. Hansen was supported by the Danish Ministry of Science Technology and Innovation.

T. P. Hansen and M. D. Nielsen are with Crystal Fiber A/S, DK-3460 Birkerød, Denmark, and with the COM Center, Technical University of Denmark, DK-2800 Kgs. Lyngby, Denmark (e-mail: tph@crystal-fiber.com).

J. Broeng, C. Jakobsen, G. Vienne, H. R. Simonsen, P. M. W. Skovgaard, and J. R. Folkenberg are with Crystal Fiber A/S, DK-3460 Birkerød, Denmark.

A. Bjarklev is with the COM Center, Technical University of Denmark, DK-2800 Kgs. Lyngby, Denmark.

Digital Object Identifier 10.1109/JLT.2003.822833 sensitivity will allow very flexible use and easy integration in supporting mechanical systems.

In this paper, we report a new design of air-guiding PBG fiber, and demonstrate guidance over a record length of $345 \mathrm{~m}$ as we first reported in [6]. We primarily focus on fibers that are optimized for guiding light at $1.55 \mu \mathrm{m}$, and investigate the spectral properties for various fiber lengths. Significant differences of the spectral characteristics are observed-demonstrating different widths of spectral transmission windows for short and long fiber lengths. We further demonstrate practical handling of air-guiding fibers for use in connection with standard optical fibers by splicing an air-guiding PBG fiber to a standard single-mode fiber. We show that loss measurements using conventional optical time domain reflectometer (OTDR) equipment are feasible, despite the nonconventional hollow fiber core. Finally, to the best of our knowledge, we present the first macrobending loss measurements on air-guiding PCFs. These measurements are performed on both a single and a multimode fiber.

\section{Fiber Design AND TRANSMission Properties}

The fiber is fabricated using a traditional stack-and-draw approach where a core defect may readily be created by removing a number of tubes from the center of the preform. Previously, cores formed from seven missing tubes have been demonstrated [1]-[3], but here we consider fibers with cores formed by removing both 7 and 19 tubes. Optical microscope pictures of the two fibers are seen in Fig. 1 (top). The pictures are reproduced to the same scale to allow easy comparison of the fibers. The fibers have core diameters of approximately $10 \mu \mathrm{m}$ (left), and $15 \mu \mathrm{m}$ (right). Furthermore, Fig. 1 (bottom) shows the recorded near-field distributions in the fibers. These observed near field distributions indicates the fiber shown on Fig. 1 (left) to be single mode while the fiber shown in Fig. 1 (right) is clearly multimode. The cladding structure of the single-mode fiber is comprised of ten rings of holes while the multimode fiber has eight rings of holes surrounding the core.

The fiber guides light due to a fundamental PBG positioned in the near-infrared wavelength range. Fig. 2(a) shows the recorded transmission spectra for $2 \mathrm{~m}$ samples of the two fibers. It is interesting to note that, while the single-mode fiber features a flat transmission peak, the transmission spectrum for the multimode fiber shows a number of peaks. These features show similarity to features in the transmission spectrum of the fiber reported in [7], where they are explained as coupling from the core mode 

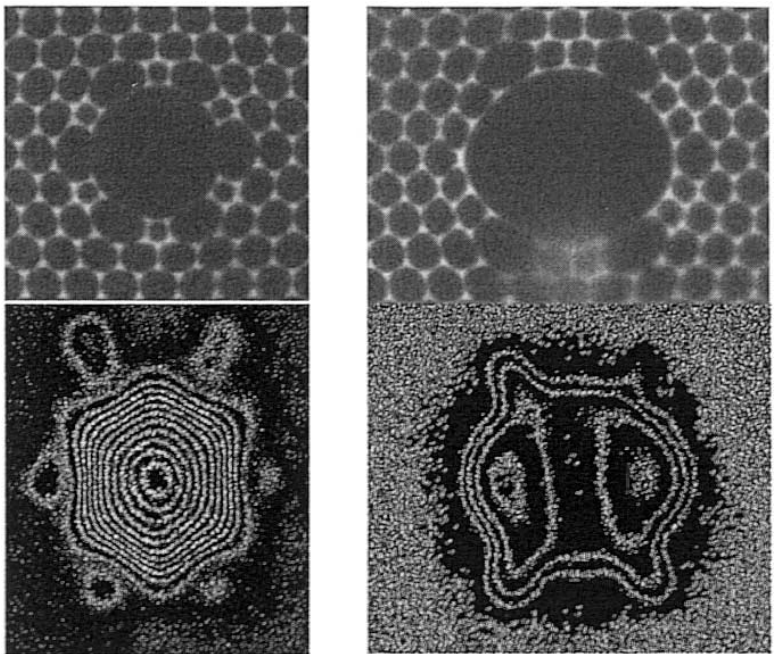

Fig. 1. Microscope (top) and near field (bottom) images of the core region of the single-mode (left) and multimode (right) fiber considered.

to index-guided surface mode in the silica surrounding the core. Note that these features also show up in the cut-back measurement presented discussed later [see Fig. 5(b)]. The exact position of the PBG may be tailored by tuning the pitch (distance between the centers of two neighboring air holes) of the photonic crystal cladding. Fig. 2(b) demonstrates fabricated fibers with a pitch of $2.5 \mu \mathrm{m}$ and $3.0 \mu \mathrm{m}$ for air-guidance around 1.30 and $1.55 \mathrm{~mm}$, respectively. In both cases, the fibers have a normalized center wavelength of $\lambda / \Lambda=1.3 / 2.5 \approx 1.55 / 3.0 \approx 0.52$. The transmission window opened by the PBG for the $1.30 \mu \mathrm{m}$ fiber is narrower, relative to the wavelength, than for the 1.55 $\mu \mathrm{m}$ fiber due to a slightly lower air-filling fraction in the fiber cladding structure. To estimate the longitudinal stability of the drawing process, we measured the transmission spectra for two $1 \mathrm{~m}$ samples of the $1.30 \mu \mathrm{m}$ fiber separated by $500 \mathrm{~m}$ in a single fiber drawing. As seen in Fig. 2(b), the spectra are seen to nearly coincide, which indicates a high degree of longitudinal uniformity.

The multimode fiber optimized for $1.55 \mu \mathrm{m}$ light was drawn in length of more than $300 \mathrm{~m}$, and Fig. 3 shows the measured transmission spectrum for fiber lengths of 2, 100, and $345 \mathrm{~m}$. From the transmission spectra, we observe a significant narrowing of the transmission bandwidth for longer fiber lengths; the 2-m PBG fiber sample has a transmission window of $\sim 200$ $\mathrm{nm}$, whereas the spectrum is seen to break up into three separate peaks for the longer fiber lengths.

\section{Fiber Handling And Attenuation Measurements}

The spectral properties of the multimode fiber were characterized using standard single-mode pigtails spliced to the PBG fiber samples. The fibers were designed with an outer diameter of $125 \mu \mathrm{m}$ that allow for easy handling and the use of conventional fiber tools, i.e., cleavers, strippers, and splicers. The excess loss caused by the fusion splice process was found to be below $0.5 \mathrm{~dB}$, by measuring the observed drop in transmission from before to after splicing (Fig. 4). Ideally, this excess loss is only caused by changes in the structure introduced by the splice process, but it may also be caused by misalignment of the fibers

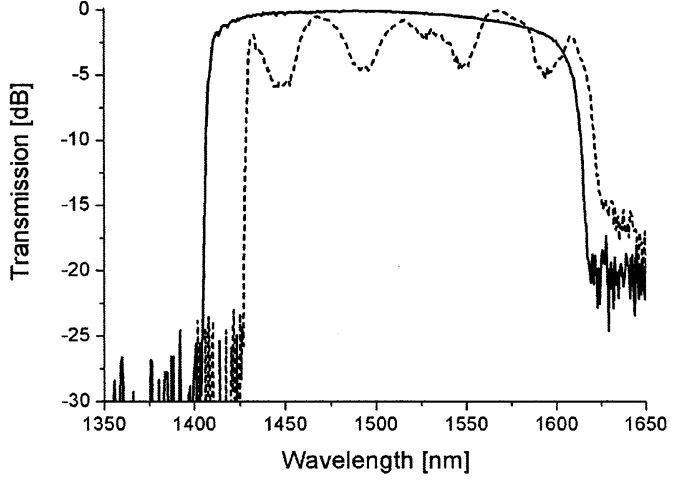

(a)

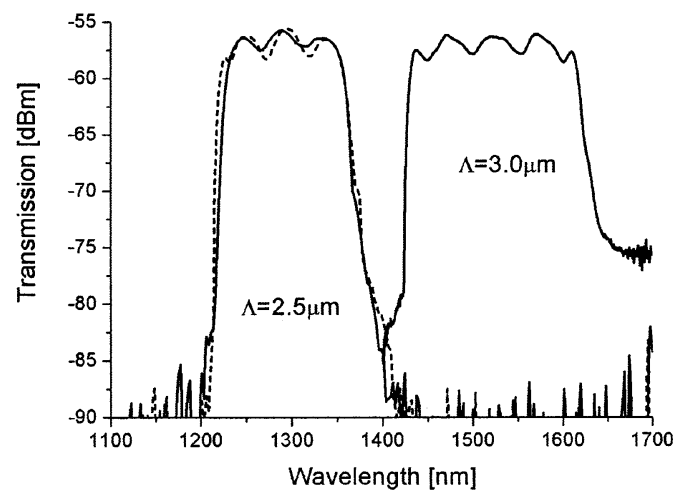

(b)

Fig. 2. (a) Transmission spectra for the single- (solid) and multimode (dashed) fiber, shown in Fig. 1. (b) Transmission spectra for two short pieces of fiber with PBGs centered at $1.30 \mu \mathrm{m}$ and $1.55 \mu \mathrm{m}$, respectively. For the $1.30-\mu \mathrm{m}$ fiber, two $1-\mathrm{m}$ samples separated by $500 \mathrm{~m}$ from the same pull are shown. The almost coinciding spectra indicate a good longitudinal stability of the fiber-drawing process.

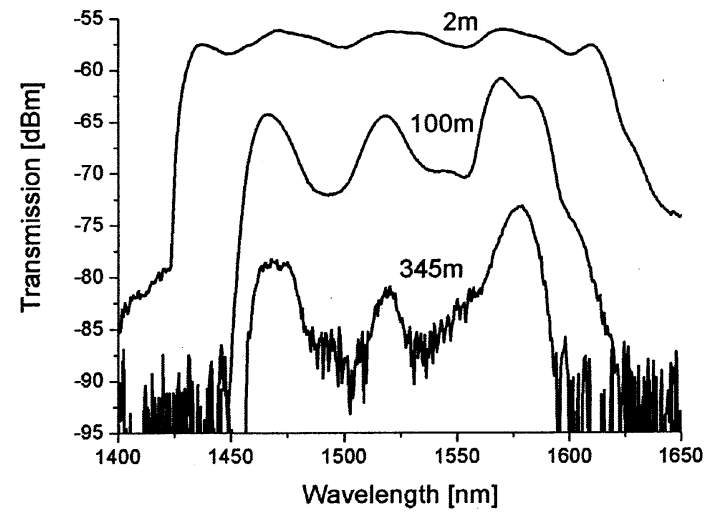

Fig. 3. Transmission spectra for fiber lengths of $2 \mathrm{~m}$ (top), $100 \mathrm{~m}$ (middle), and $345 \mathrm{~m}$ (bottom).

introduced by the splicer during the process. The splice loss is primarily given by the mode mismatching between the two dissimilar fibers joined together. Fresnel reflection at the glass-air interface adds $0.2 \mathrm{~dB}$ to the splice loss. It should be noted, that the splice-procedure used may be further optimized to lower the deformation-induced loss.

We have further studied the air-guiding fibers when used with equipment developed for standard optical fiber technology. 


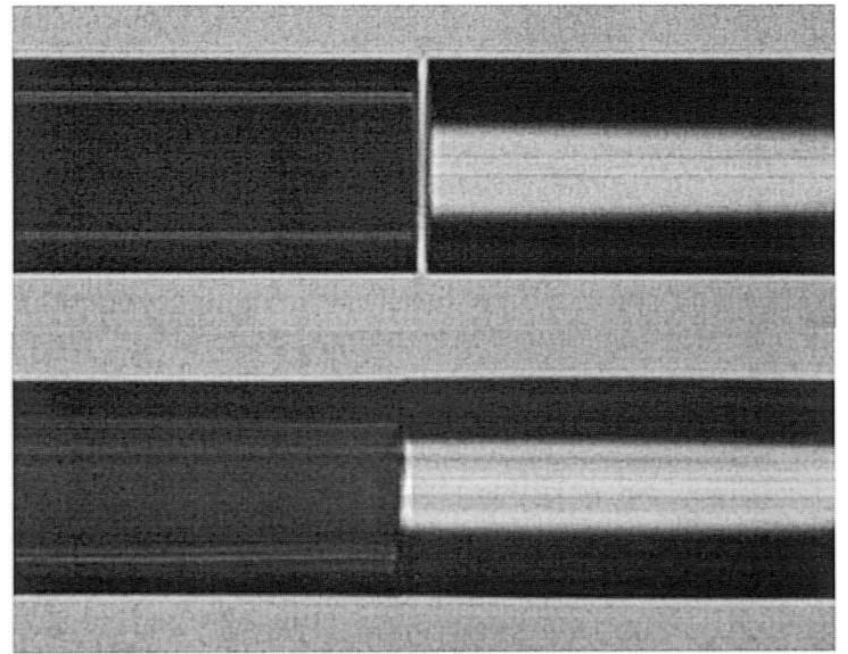

Fig. 4. The air-guiding fiber (left) fusion spliced to a standard single-mode pigtail (right)

OTDR is a well-established procedure for measuring longitudinal loss profiles of optical fibers. As shown in Fig. 5(a), air-guiding fiber may be characterized using OTDR. The measured back-scattered signal is significantly higher than expected from Rayleigh scattering in the air and may arguably be due to back scattering of light by roughness on the air/silica interface around the core. For the setup, we found it necessary to reduce the Fresnel back-reflection from the interface between the OTDR connection fiber and the PBG fiber, to avoid saturating the detector. This was realized by butt coupling the air-guiding fiber to an FC/APC connected standard single-mode fiber. The obtained OTDR trace of a 100-m multimode air-guiding fiber is shown in Fig. 5(a). When performing OTDR measurements, the recorded time-of-flight of the back-scattered pulse is translated to distance through knowledge of the mode index of the guided mode. An important verification of the results shown here is that the mode index must be assumed to be 1.0 (corresponding to light in air) in order to match the measured position of the observed reflection from the fiber end with the physical fiber length. At a wavelength of $1.55 \mu \mathrm{m}$, the trace is shown both with and without an external metal reflector at the output facet. Without the reflector, no back scattering is observed from the fiber end, in contrast to the case for conventional fibers. In such fibers a reflection from the output end would be observed due to Fresnel reflection on the glass/air interface. Also shown are the responses when only the connector fiber is present, and the OTDR trace of the connected PBG fiber obtained at $1.31 \mu \mathrm{m}$, i.e., outside the PBG. In both cases, a rapidly decaying signal is observed. The attenuation obtained from the OTDR trace of approximately $80 \mathrm{~dB} / \mathrm{km}$. To verify this loss level, the spectral fiber attenuation of the $100 \mathrm{~m}$ fiber was also measured using the cut-back technique. Fig. 5(b) shows the resulting spectral loss curve. The minimum loss observed is $61 \mathrm{~dB} / \mathrm{km}$ at a wavelength of $1570 \mathrm{~nm}$. At $1550 \mathrm{~nm}$ the loss is approximately $125 \mathrm{~dB} / \mathrm{km}$. Observing this steep loss edge around $1550 \mathrm{~nm}$, the $80 \mathrm{~dB} / \mathrm{km}$ predicted by the OTDR measurement must be considered in agreement with the results of the cut-back measurement. This indicates that OTDR may very well prove

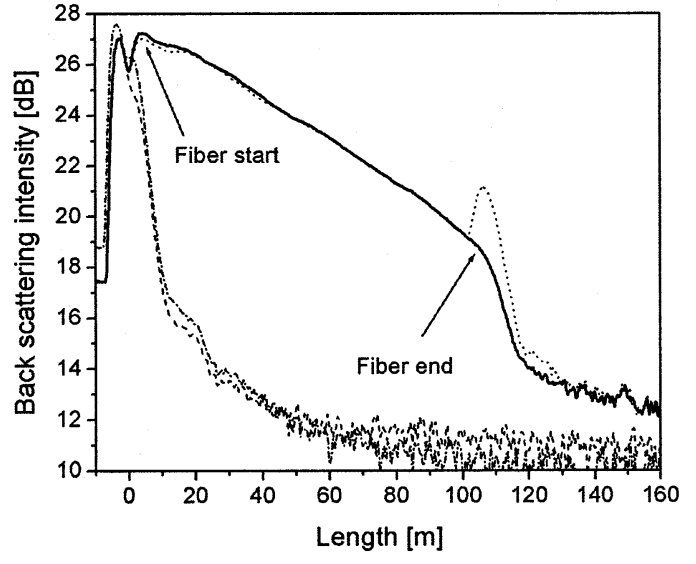

(a)

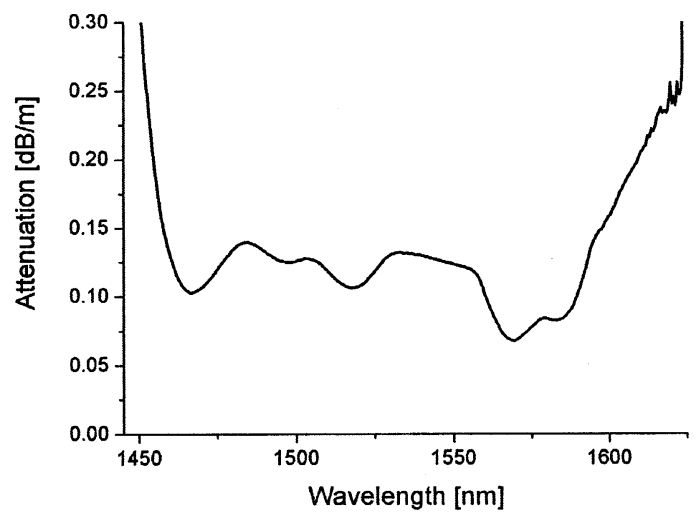

(b)

Fig. 5. (a) OTDR trace of $100 \mathrm{~m}$ of air-guiding fiber (between arrows). Fiber alone at a wavelength of $1.55 \mu \mathrm{m}$ (full line), and with external reflector at the output facet (dotted). For comparison, the fiber with external reflector at 1.31 $\mu \mathrm{m}$ (dashed), and the connector cable with no air-guide present (dash-dot). (b) Spectral attenuation measured by cut-back on the $100 \mathrm{~m}$ fiber.

to be as important a tool when characterizing air-guiding fibers as it is for conventional fibers.

\section{Macrobending Loss Measurements}

To measure the macrobending loss, first a straight-fiber reference measurement was performed. These reference measurements are the ones shown above in Fig. 2(a). While the coupling conditions were maintained, the fibers were then subject to different degrees of bending. Bends of $40 \mathrm{~mm}$ (10 turns), $20 \mathrm{~mm}$ (1 turn), $10 \mathrm{~mm}$ (10 turns), and $4 \mathrm{~mm}$ (1 turn) were studied. A splice to a standard single mode fiber pigtail was used to give stable coupling conditions to the single mode fiber. In Fig. 6(a), recorded transmission spectra for a number of bending diameters are shown for the single-mode PBG fiber. Some bending-induced changes are observed in the figure at the short wavelength transmission window edge, but over most of the bandwidth no change is seen. To quantify these changes we calculated the bending loss as the difference in transmission between the straight fiber reference and the bent fiber measurement. The bending loss for the single mode fiber is shown in Fig. 6(b). Except for the short wavelength edge, the measured bending loss 


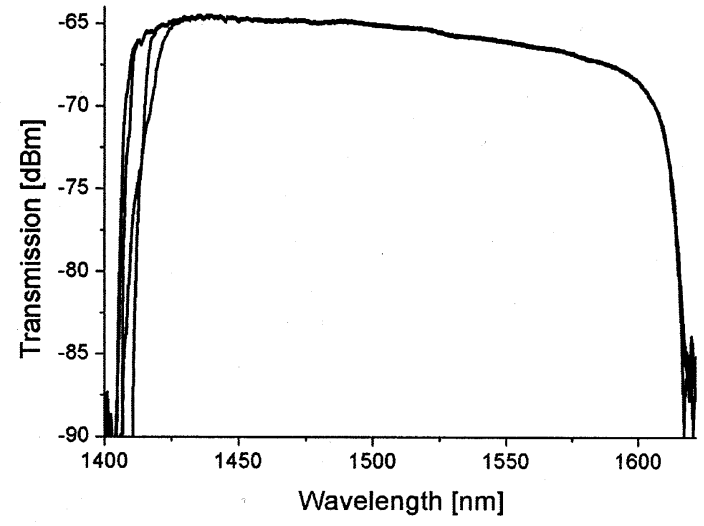

(a)

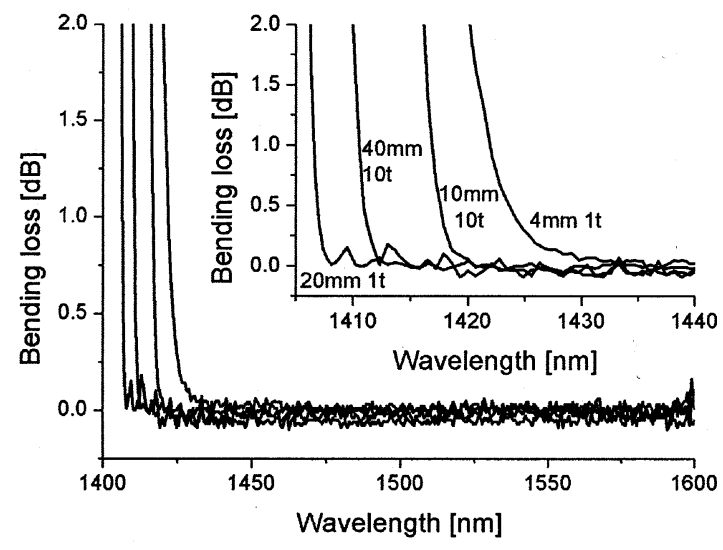

(b)

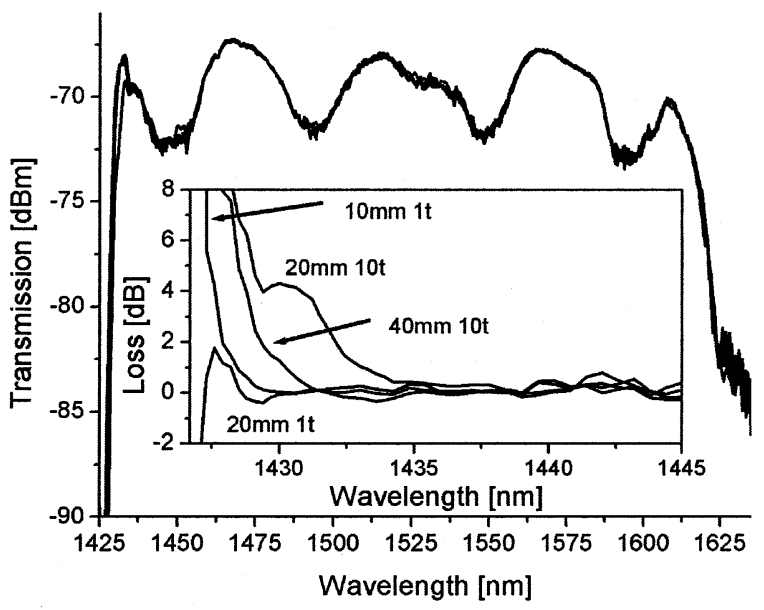

(c)

Fig. 6. (a) Transmission spectra, (b) loss measurements for the single-mode fiber, and (c) transmission spectra for the multimode fiber. Inset in (c) shows the loss measurements for the multimode fiber. The bending diameters used are indicated on the insets.

is $0 \mathrm{~dB} \pm 0.1 \mathrm{~dB}$. The inset shows the loss at the short wavelength edge. It is seen that a 1 turn bend with a diameter of $4 \mathrm{~mm}$ shifts the $1 \mathrm{~dB}$ loss point $15 \mathrm{~nm}$ compared to 1 turn with $20 \mathrm{~mm}$ of diameter, which is seen to give the lowest loss. Maintaining a bending diameter this small for prolonged periods makes the fiber likely to break. Therefore, the fiber is concluded to be insensitive to macrobending at all practical diameters.

Fig. 6(c) shows the transmission spectra measured for the multimode fiber. The fiber was subject to $40 \mathrm{~mm}$ (10 turns), 20 $\mathrm{mm}$ (10 turns and 1 turn), and $10 \mathrm{~mm}$ (1 turn) bends. For this measurement, the in coupling was performed by a butt coupling to a single-mode fiber. The same qualitative behavior as for the single mode fiber is seen. Only at the short wavelength transmission band edge is the bending seen to induce loss. The accuracy of the measurements depends on the position in the bandgap. At a local maximum, the transmission spectra are in excellent agreement, within $0.2 \mathrm{~dB}$, while rapid fluctuations are seen at the local minima. As the transmission minima are caused by coupling between the core mode and surface modes, a process that is highly sensitive to environmental changes to the fiber, such fluctuations are expected. The inset in Fig. 6(c) shows the bending induced loss in the multimode fiber, analogous to the inset in Fig. 6(b). The curve seen to disappear in the negative direction at short wavelengths is measured for single turn and 20 $\mathrm{mm}$ bend diameter. This unphysical behavior is caused by the short-wavelength bandgap edge observed in the reference measurement.

That both fibers are seen to be insensitive to bending over most of the bandgap may be understood from mode index considerations. From [8, Fig. 2], the step between the core index (1.0) and the bandgap edge, corresponding to the cladding index, may be estimated to $2 \cdot 10^{-2}$. This step between core and cladding index is significantly larger than what is found in conventional solid fibers but may be obtained in index-guiding PCFs. This very tight confinement of the guided-mode results in the robust guiding even through tightly bended fibers.

\section{CONCLUSION}

We have demonstrated transmission of IR-light through a record length of $345 \mathrm{~m}$ large-core air-guiding PBG fiber. We have resolved spectral features of the PBG region, and for the first time, reported splicing of air-guiding fiber to standard fiber. The fiber attenuation was measured using conventional OTDR equipment and verified by cut-back measurements. Finally, macrobending loss was measured for two different fibers. Both fibers were found to be insensitive to macrobending over the majority of the bandgap.

\section{REFERENCES}

[1] R. F. Cregan, B. J. Mangan, J. C. Knight, T. A. Birks, P. St. J. Russell, P. J. Roberts, and D. C. Allan, "Single-mode photonic band gap guidance of light in air," Science, vol. 285, pp. 1537-1539, 1999.

[2] J. B. West, J. C. Fajardo, M. T. Gallagher, K. W. Koch, N. F. Borrelli, and D. C. Allan, "Demonstration of an IR-optimized air-core photonic band-gap fiber," in ECOC 2000 Proc., Munich, Germany, 2000, paper no. 10.2 .3 .

[3] N. Venkataraman, M. T. Gallagher, C. M. Smith, D. Müller, J. A. West, K. W. Koch, and J. C. Fajardo, "Low loss $(13 \mathrm{~dB} / \mathrm{km})$ air core photonic band-gap fiber," in ECOC 2002 Proc., Copenhagen, Denmark, 2002, post-deadline paper no. PD1.1.

[4] T. Sorensen, J. Broeng, A. Bjarklev, E. Knudsen, and S. E. B. Libori, "Macro-bending loss properties of photonic crystal fiber," Electron. Lett., vol. 37, pp. 287-289, 2001.

[5] E. Knudsen, A. Bjarklev, J. Broeng, and S. E. Barkou, "Macro-bending loss estimation for air-guiding photonic crystal fibers," in OFS 2000, 14th Int. Conf. Optical Fiber Sensors, 2000, paper FR 4-4, pp. 904-907. 
[6] T. P. Hansen, J. Broeng, C. Jakobsen, G. Vienne, H. R. Simonsen, M. D. Nielsen, P. M. W. Skovgaard, J. R. Folkenberg, and A. Bjarklev, "Airguidance over 345 m large-core photonic bandgap fiber," in OFC 2003 Proc., Atlanta, GA, 2003, post-deadline paper PD4-1.

[7] C. M. Smith, N. Venkataraman, M. T. Gallagher, D. Müller, J. A. West, N. F. Borrelli, D. C. Allan, and K. W. Koch, "Low-loss hollow-core silica/air photonic bandgap fiber," Nature, vol. 424, pp. 657-659, 2003.

[8] J. Broeng, S. E. Barkou, T. Sondergaard, and A. Bjarklev, "Analysis of air-guiding photonic bandgap fibers," Opt. Lett., vol. 25, pp. 96-98, 2000

Theis P. Hansen, photograph and biography not available at the time of publication.

Jes Broeng, photograph and biography not available at the time of publication.

Christian Jakobsen, photograph and biography not available at the time of publication.
Guillaume Vienne, photograph and biography not available at the time of publication.

Harald R. Simonsen, photograph and biography not available at the time of publication.

Martin D. Nielsen, photograph and biography not available at the time of publication.

Peter M. W. Skovgaard, photograph and biography not available at the time of publication.

Jacob R. Folkenberg, photograph and biography not available at the time of publication.

Anders Bjarklev, photograph and biography not available at the time of publication. 